\title{
ARTICLE \\ Sympathetic transmitters control thermogenic efficacy of brown adipocytes by modulating mitochondrial complex $\mathrm{V}$
}

\author{
Tao-Rong $\mathrm{Xie}^{1,3}$, Chun-Feng Liu ${ }^{1,2,3}$ and Jian-Sheng Kang ${ }^{1}$
}

Obesity is a worldwide epidemic and results from excessive energy intake or inefficient energy expenditure. It is promising to utilize the thermogenic function of brown adipose tissue for obesity intervention. However, the mechanisms controlling the efficacy of norepinephrine-induced thermogenesis in brown adipocytes remain elusive. Here we demonstrate that norepinephrine (NE) induces low-efficacy thermogenesis, evoking both heterogeneous changes $\left(\Delta \Psi_{\mathrm{m}}\right.$ and $\left.\Delta \mathrm{pH}\right)$ and homogenous responses, one of which is that NE stimulation causes large amounts of ATP consumption in brown adipocytes. We reveal that the protonATPase activity of mitochondrial complex $\mathrm{V}$ is a key factor that antagonizes proton leakage by UCP1 and determines the efficacy of NE-induced thermogenesis in brown adipocytes. Furthermore, to avoid unnecessary and undesired heat production, we reveal that ATP is a necessary sympathetic cotransmitter for the high efficacy and specificity of NE-induced thermogenesis in brown adipocytes as it increases intracellular calcium concentrations and upregulates the ATP synthase activity of complex V. Thus, we demonstrate the modulation mechanism of thermogenic efficacy in brown adipocytes. These findings imply new strategies to partially or fully utilize the thermogenic capacity of brown adipocytes to identify therapeutic targets for the treatment of obesity and diabetes.

Signal Transduction and Targeted Therapy (2017) 2, e17060; doi:10.1038/sigtrans.2017.60; published online 10 November 2017

\section{INTRODUCTION}

Obesity is a worldwide epidemic and results from excessive energy intake or inefficient energy expenditure. Brown adipose tissue (BAT) is the major tissue for cold-induced thermogenesis (energy dissipation as heat) without shivering. ${ }^{1,2}$ The identification of functional BAT in adult humans ${ }^{3-5}$ suggests that it is promising to utilize the thermogenic capacity of BAT for obesity and diabetes treatments. ${ }^{6,7}$

Currently, the mechanism of thermogenesis in BAT and brown adipocytes (BA) is known in general. ${ }^{2}$ Cutaneous thermosensory signals for cold stimulation evoke sympathetic nerve firing in BAT via the hypothalamus. ${ }^{8}$ The released neurotransmitter norepinephrine (NE) binds the adrenoreceptors of BA, upregulates hormone-sensitive lipase (HSL)-mediated lipolysis via phosphorylation by protein kinase $A$ signaling, mobilizes free fatty acids to activate mitochondrial uncoupling protein-1 (UCP1) and converts electrochemical potential energy stored in the mitochondrial proton gradient to heat. ${ }^{2,9}$ However, our previous study demonstrates the low thermogenic efficacy of NE-induced thermogenesis in $\mathrm{BA}^{10}$ which may partially account for the failures of trials utilizing the thermogenic capacity of BAT for obesity intervention. ${ }^{11,12}$ Therefore, the regulatory mechanism of thermogenesis in BA is still largely unknown. In this study, we demonstrate that the proton-ATPase function of mitochondrial complex $\mathrm{V}$ accounts for $\mathrm{NE}$-induced heterogeneous changes in $\mathrm{BA}$ and that sympathetic cotransmitter ATP enhances the efficacy of thermogenesis in BA.

\section{MATERIALS AND METHODS}

Data are pooled from or repeated for each condition with at least three independent experiments. No statistical methods were used to predetermine the sample size. All data points in the figures represent mean \pm s.e.m., except for Figure $3 e$ in which data in the text or figures represent mean \pm s.d. More detailed information on the experimental procedures can be found in the Supplementary Data.

Isolation and primary culture of brown adipocytes

$\mathrm{BA}$ were isolated from 3- to 4-week-old male $\mathrm{C} 57 \mathrm{BL} / 6 \mathrm{~J}$ mice with a procedure similar to that described by Lucero et al. ${ }^{13}$ Briefly, mice were kept at $4{ }^{\circ} \mathrm{C}$ overnight with free access to food and water to deplete stored lipids in BAT. The mice were then killed by cervical dislocation and swabbed with $75 \%$ ethanol. Interscapular BAT was isolated and placed in an isolation buffer (DMEM with $4 \%$ NCS). The tissue was minced and digested with $0.2 \%$ collagenase type II in a shaking water bath at $37^{\circ} \mathrm{C}$ for 30 min. $^{14}$ After digestion, the reaction mixture was discarded and the tissue was washed with isolation buffer. Cells were dissociated by gently triturating with fire-polish pipettes and washed by centrifugation in PBS. After the final washing, the cells were plated onto 12-mm coverslips $\left(\sim 3 \times 10^{4}\right.$ cells per coverslip). Coverslips were precoated with Matrigel. After $2 \mathrm{~h}, 2 \mathrm{ml}$ of plating medium (DMEM supplemented with $5 \% \mathrm{FBS}$, 100 units per $\mathrm{ml}$ penicillin and $100 \mathrm{\mu g} \mathrm{ml}^{-1}$ streptomycin) was added to each $35-\mathrm{mm}$ dish. From the second day in culture onward, half of the medium was replaced with feeding medium (plating medium supplemented with Ara-C to inhibit fibroblast proliferation and $2 \mu \mathrm{M}$ final concentration of Ara-C) every 2 days. Cells were maintained at $37^{\circ} \mathrm{C}$ in a humidified atmosphere of $95 \%$ air and $5 \% \mathrm{CO}_{2}$ and used for imaging at 3-8 days in vitro.

\footnotetext{
${ }^{1}$ CAS Key Laboratory of Nutrition and Metabolism, Institute for Nutritional Sciences, Shanghai Institutes for Biological Sciences, Chinese Academy of Sciences, Shanghai, China and ${ }^{2}$ Technical Center for Animal, Plant and Food Inspection and Quarantine, Shanghai Entry-Exit Inspection and Quarantine Bureau, Shanghai, China.

Correspondence: J-S Kang (jskang@gmail.com)

${ }^{3}$ These authors contributed equally to this work.

Received 12 July 2017; revised 10 September 2017; accepted 26 September 2017
} 


\section{Cell transfection}

$\mathrm{BA}$ were washed with $\mathrm{PBS}$ and resuspended in electroporation solution (20 mM Hepes, $135 \mathrm{mM} \mathrm{KCl}, 2 \mathrm{mM} \mathrm{MgCl}$, 0.5\% Ficoll 400, 1\% DMSO, 2 mM ATP and $5 \mathrm{mM}$ glutathione, $\mathrm{pH}$ 7.6) with $20 \mu \mathrm{g}$ of plasmid DNA. ${ }^{15}$ Two pulses (115V, 10-ms duration) with 1-s intervals were delivered with an ECM 830 Square Wave Electroporation System (Harvard Apparatus, Inc., Holliston, MA, USA) to electroporate cells $\left(1-1.5 \times 10^{6}\right.$ cells per $\left.\mathrm{ml}\right)$. Then, cells were seeded onto Matrigel-coated coverslips. BA were maintained in DMEM supplemented with 5\% FBS until imaging.

\section{Thermogenic study}

All imaging was performed using a confocal microscope with a $40 \times / 0.95$ objective (Olympus, Shinjuku, Japan) for time-lapse imaging and a $100 \times /$ 1.40 objective (Olympus) for high-resolution imaging. Cells were costained with RhB-ME and Rh800 ( $20 \mathrm{nM}$ dyes for time-lapse imaging and $50 \mathrm{nM}$ dyes for high-resolution imaging) in Tyrode's solution (in $\mathrm{mM}: 10$ Hepes, 10 glucose, $3 \mathrm{KCl}, 145 \mathrm{NaCl}, 1.2 \mathrm{CaCl}_{2}$, and $1.2 \mathrm{MgCl}_{2}, \mathrm{pH} 7.4$ ) for $1 \mathrm{~h}$ at $33^{\circ} \mathrm{C}^{10}$ The pseudocolor of the RhB-ME channel is red (excited at $559 \mathrm{~nm}$ and collected at $575-620 \mathrm{~nm}$ ), and the Rh800 channel is green (excited at $635 \mathrm{~nm}$ and collected at $655-755 \mathrm{~nm}$ ). All images were collected at $512 \times 512$ (for time-lapse imaging) and $1600 \times 1600$ (for highresolution imaging) pixel resolutions (12 bit).

To minimize the heat influence of the perfusion solution, time-lapse imaging of BA was performed in $2 \mathrm{ml}$ of Tyrode's solution rather than in the perfusion system. To minimize bleaching and damage to live BA in time-lapse imaging, the lowest intensity of lasers with the largest pinhole setting and the shortest scanning time were used, and 101 frames were recorded in time-lapse imaging at 30-s intervals. NE $(0.1 \mu \mathrm{M})$, ATP $(10 \mu \mathrm{M})$ or vehicle was injected as early as the 11th frame of time-lapse imaging for the thermogenesis studies in BA. SR-59230 A $(1 \mu \mathrm{M})$ or oligomycin $\left(10 \mu \mathrm{g} \mathrm{ml}^{-1}\right)$ was injected $15 \mathrm{~min}$ before the time-lapse imaging in pharmacological tests.

After background was removed, ratiometric values of the Rh800 channel to the RhB-ME channel (simultaneously excited by $635-\mathrm{nm}$ and $559-\mathrm{nm}$ lasers) were calculated pixel by pixel to represent the thermal response of the sample. For noise reduction, the pixels with signal-to-noise ratios less than 1.5 were excluded, and a $5 \times 5$ moving average was used before the ratiometric process. According to the three-sigma rule, the outlier $(99.7 \%$ tolerance interval) of the ratios was also excluded. The ratio of each cell was the average ratio of all pixels representing the cell. Since we focused on thermal responses, the mean ratio of the steady state before drug treatments was used to normalize every data point for each cell. All data analysis was performed with MATLAB (MathWorks Inc., Natick, MA, USA) and ImageJ (NIH, Bethesda, MD, USA).

\section{Cytoplasmic pH study}

Cytoplasmic $\mathrm{pH}$ imaging was performed using a confocal microscope with a $40 \times / 0.95$ objective (Olympus) for time-lapse imaging. Cells were costained with $5 \mu \mathrm{M}$ SNARF1-AM and $20 \mathrm{nM}$ Rh800 in Tyrode's solution for $30 \mathrm{~min}$ at $37^{\circ} \mathrm{C}$. Then, SNARF1-AM was washed out using Tyrode's solution (with $20 \mathrm{nM}$ Rh800). The pseudocolor of the acidification channel is red (excited by 561-nm laser and collected with 575-600-nm bandpass filter), the alkalization channel is green (simultaneously collected with 600-675$\mathrm{nm}$ bandpass filter), and the Rh800 channel is magenta (sequentially excited by $635-\mathrm{nm}$ laser and collected with $655-755-\mathrm{nm}$ bandpass filter). Ratiometric values of the acidification channel to the alkalization channel were calculated pixel by pixel to represent the relative $\mathrm{pH}$ value of the sample. The other conditions of imaging, data acquisition and analysis for the cytoplasmic pH study were the same as the conditions in the thermogenic study.

\section{Redox (FAD/FAD+NADH) measurement}

Redox (FAD/FAD+NADH) imaging was performed using a customized fluorescence microscope with a $40 \times / 0.8 \mathrm{~W}$ objective (Olympus) for timelapse imaging. The endogenous autofluorescence images ${ }^{16}$ were excited with an Optoscan monochromator (Cairn Research Ltd., Kent, UK). The pseudocolor of the FAD channel is red (excited by 430-nm light with 20$\mathrm{nm}$ bandwidth and collected with 525-575-nm bandpass filter), whereas the NADH+FAD channel is green (sequentially excited by $340-\mathrm{nm}$ light with 20-nm bandwidth and collected with 420-nm long pass filter). In addition, the fluorescence images were acquired with an Evolve 512 EMCCD (Photometrics Ltd., Tucson, UK). Time-lapse imaging of BA was performed in $4 \mathrm{ml}$ of Tyrode's solution at $33^{\circ} \mathrm{C}$. Sixty frames were recorded in a time-lapse imaging at 30-s intervals. NE $(0.1 \mu \mathrm{M})$ was injected as early as the 11th frame of time-lapse imaging for the redox studies in BA. The other conditions of imaging, data acquisition and analysis for the redox study were the same as the conditions in the thermogenic study.

\section{Cytoplasmic $\left[\mathrm{Ca}^{2+}\right]$ study}

Cytoplasmic $\left[\mathrm{Ca}^{2+}\right]$ imaging was performed using the customized fluorescence microscope with a $40 \times / 0.8 \mathrm{~W}$ objective (Olympus) for timelapse imaging and an Optoscan monochromator (Cairn Research Ltd.) as a light source. Cells were stained with $5 \mu$ M Fura2-AM in Tyrode's solution for $30 \mathrm{~min}$ at $37^{\circ} \mathrm{C}$. Then, Fura2-AM was washed out using Tyrode's solution. The pseudocolor of the 380-nm channel is red (excited by 380-nm light with 10-nm bandwidth and collected with $505-535-\mathrm{nm}$ bandpass filter), and the $340-\mathrm{nm}$ channel is green (sequentially excited by $340-\mathrm{nm}$ light with 10-nm bandwidth and collected with 505-535-nm bandpass filter). Ratiometric values of the $340-\mathrm{nm}$ channel to the $380-\mathrm{nm}$ channel were calculated pixel by pixel to represent the relative $\left[\mathrm{Ca}^{2+}\right]$ of the sample. The other conditions of imaging, data acquisition and analysis for the cytoplasmic $\left[\mathrm{Ca}^{2+}\right]$ study were the same as the conditions in the redox study.

\section{Mitochondrial $\left[\mathrm{Ca}^{2+}\right]$ study}

Mitochondrial $\left[\mathrm{Ca}^{2+}\right]$ imaging was performed using a confocal microscope with a $40 \times / 0.95$ objective (Olympus) for time-lapse imaging. Cells were transfected with 4mtD3cpv (from Dr R Tsien) using an ECM 830 Square Wave Electroporation System (Harvard Apparatus, Inc., USA). The pseudocolor of the CFP channel is cyan (excited by 440-nm laser and collected with 480-495-nm bandwidth filter) and the YFP channel is magenta (simultaneously collected with 505-605-nm bandwidth filter). Ratiometric values of the YFP channel to the CFP channel were calculated pixel by pixel to represent the relative $\left[\mathrm{Ca}^{2+}\right]$ of the sample. The other conditions of imaging, data acquisition and analysis for the mitochondrial $\left[\mathrm{Ca}^{2+}\right]$ study were the same as the conditions in the cytoplasmic $\left[\mathrm{Ca}^{2+}\right]$ study.

\section{Cytoplasmic and mitochondrial [ATP] study}

[ATP] imaging was performed using a confocal microscope with a $40 \times /$ 0.95 objective (Olympus) for time-lapse imaging. Cells were transfected with AT1.03 for cytoplasmic [ATP] or mitAT1.03 for mitochondrial [ATP] (from $\mathrm{Dr} \mathrm{H}$ Noji and $\mathrm{Dr} \mathrm{H}$ Imamura) using an ECM 830 Square Wave Electroporation System (Harvard Apparatus, Inc.). The pseudocolor of the CFP channel is cyan (excited at $440 \mathrm{~nm}$ and collected at $480-495 \mathrm{~nm}$ ), and the YFP channel is magenta (simultaneously excited at $440 \mathrm{~nm}$ and collected at 505-605 nm). Ratiometric values of the YFP channel to the CFP channel were calculated pixel by pixel to represent the relative [ATP] of the sample. The other conditions of imaging, data acquisition and analysis for the $[A T P]$ study were the same as the conditions in the thermogenic study.

\section{RESULTS}

$\mathrm{NE}$ induces heterogeneous responses in $\mathrm{BA}$

We consistently observed that NE-induced heterogeneous changes in mitochondrial membrane potentials (MMP) in BA (Figure 1), which was monitored with thermoneutral rhodamine 800 (Rh800, a thermal-insensitive mitochondrial marker and MMP sensor). ${ }^{10,17}$ Obviously, there are two MMP populations (depolarization and hyperpolarization) of NE-induced thermogenesis in BA (Figures $1 a-h)$, which is in line with previous studies. ${ }^{10,18}$ The subpopulation of MMP depolarization can be explained by the activation of UCP1, which dissipates the electrochemical potential energy of protons as heat. The diverse extent of MMP depolarization evoked by NE may be explained by the heterogeneity and different NE affinities of the $\beta$ adrenergic receptors ${ }^{19}$ or the heterogeneous levels of UCP1 expression. ${ }^{20}$

To test this explanation, we evaluated the potential relationship between the heterogeneity of MMP changes and the extent of HSL phosphorylation at Ser563 (Supplementary Figure 1), as HSL serves as a downstream lipase of $\beta$-adrenergic receptors. After NE stimulation and monitoring of the responses of MMP, we 
a

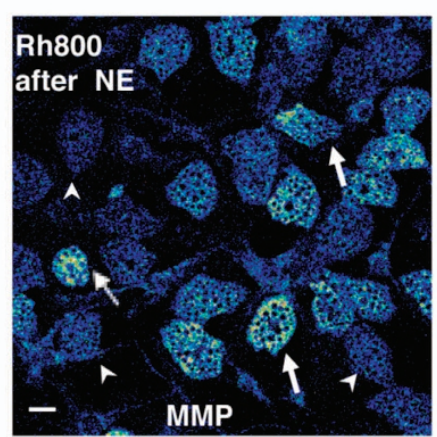

b

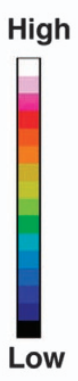

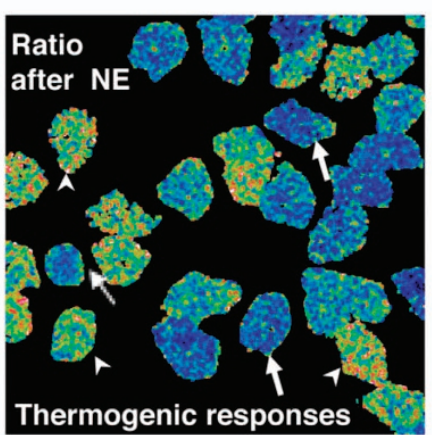

C

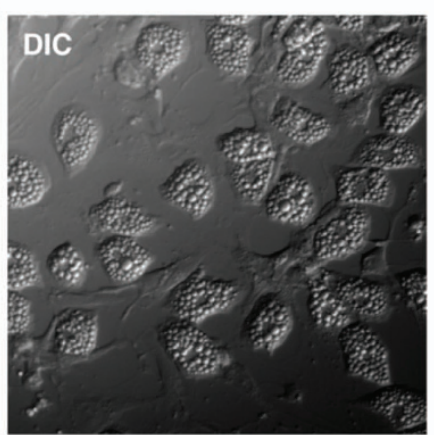

d

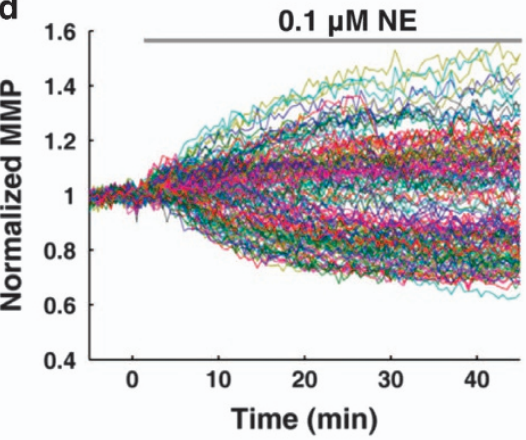

g

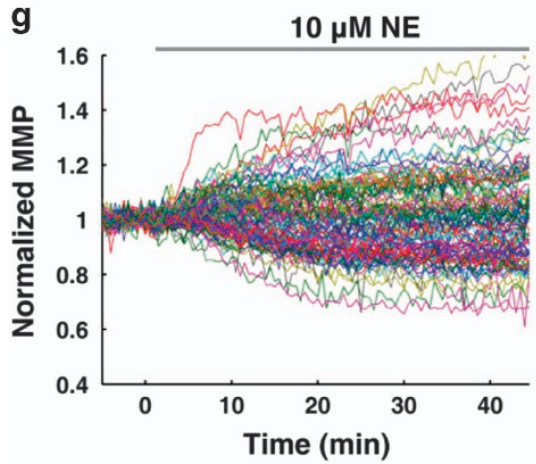

e

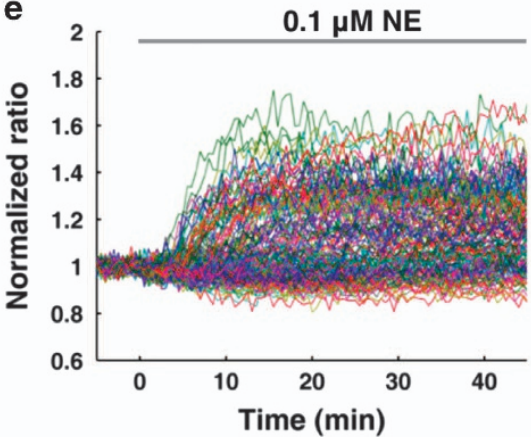

h

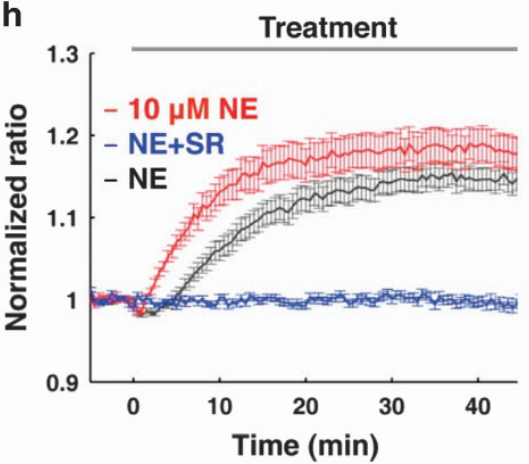

f

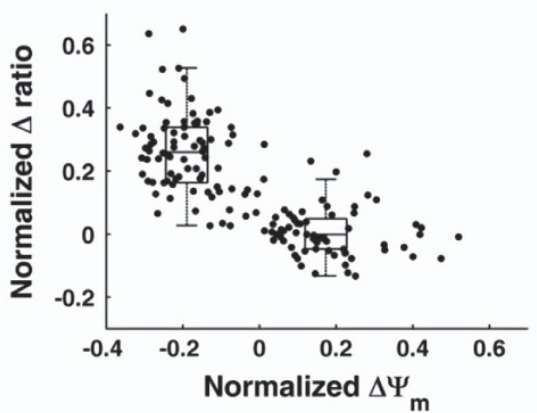

i

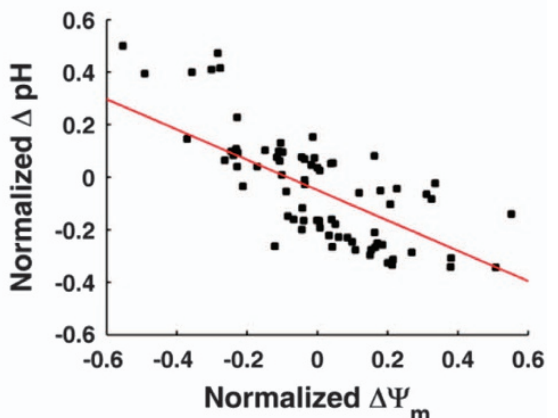

Figure 1. NE induces heterogeneous responses in BA. (a-f) NE-induced thermogenic responses in $B A$ show a negative correlation with $\Delta \Psi_{m}$. (a) Representative MMP image of BA after $0.1 \mu \mathrm{M}$ NE treatment. Arrow heads point to BA with mitochondrial depolarization, whereas arrows point to BA with mitochondrial hyperpolarization. Scale bar, $20 \mu \mathrm{m}$. (b) The corresponding thermal image (ratio of Rh800 intensity to RhB-ME intensity) after $0.1 \mu \mathrm{M}$ NE treatment. The result shows the low efficacy of NE-induced thermogenic responses in BA. Some BA show thermogenic responses to NE (arrow heads), whereas others have no response to NE stimulation (arrows). (c) Differential interference contrast (DIC) image of BA. (d) The raw data plots of MMP show subpopulations of depolarization and hyperpolarization in $0.1 \mu \mathrm{M}$ NE-treated BA. Each colored trace represents a MMP change of a single BA $(n=150)$. (e) NE-induced thermal responses in BA. Each colored trace represents a thermogenic response of a single BA $(n=150)$. (f) The scatter plot of thermogenic responses (d) versus MMP changes (e) induced by $\mathrm{NE}$, which shows a negative correlation $\left(r=-0.73, n=150\right.$, and $P=2.97 \times 10^{-26}$ by Pearson's correlation coefficient test). The box plots of the depolarization subpopulation and the hyperpolarization subpopulation show significantly different amplitudes of thermogenic responses $\left(P=1.88 \times 10^{-27}\right.$, two-sided $t$-test). (g) The raw data plots of MMP still show two-subpopulations in $10 \mu \mathrm{M}$ NE-treated BA. Each colored trace represents an MMP change of a single BA $(n=102)$. (h) Thermogenic responses in BA are evoked by $0.1 \mu \mathrm{M}$ NE (black line, $n=150$ ) and $10 \mu \mathrm{M} \mathrm{NE}$ (red line, $n=88$ ), respectively. The blue line shows that the NE-induced thermogenic response of BA is inhibited by $1 \mu \mathrm{M}$ SR-59230 A $(n=97)$. All data points in figures represent mean \pm s.e.m. (i) A representative scatter plot of pH changes versus MMP changes induced by $0.1 \mu \mathrm{M} \mathrm{NE}$, which shows a strong negative correlation (red line, $r=-0.67, n=75$, and $P=3.94 \times 10^{-11}$ by Pearson's correlation coefficient test).

retrospectively immunostained $\mathrm{BA}$ with an antibody against phospho-HSL and assumed that BA with high levels of phosphorylated HSL should correlate with mitochondrial depolarization. However, BA with high levels of phosphorylated HSL (arrows and red arrow head) either show mitochondrial depolarization (arrow heads) or hyperpolarization (arrows), whereas BA with low levels of phosphorylated HSL (white arrow heads) can show mitochondrial depolarization (Supplementary Figures 1b, c and f).
Second, treatments with 100-fold higher NE concentrations $(10 \mu \mathrm{M})$ still showed two MMP populations (Figure 1g). These results suggest that the heterogeneous changes of MMP are unlikely to be correlated to the heterogeneity of adrenoreceptors or different activities of HSL. In addition, we also found that all BA express UCP1, but it is expressed at heterogeneous levels (Supplementary Figures 2a-d), and that mitochondrial hyperpolarization is not due to the absence of UCP1 (Supplementary 
Figures 2e-g). These results suggest that the heterogeneities of p-HSL or UCP1 may only partially account for the diverse responses of mitochondrial MMP.
Correlations among NE-induced heterogeneous responses in $\mathrm{BA}$ With thermosensitive rhodamine $B$ methyl ester (RhB-ME)-based mito-thermometry, ${ }^{10}$ we observed that the NE-induced
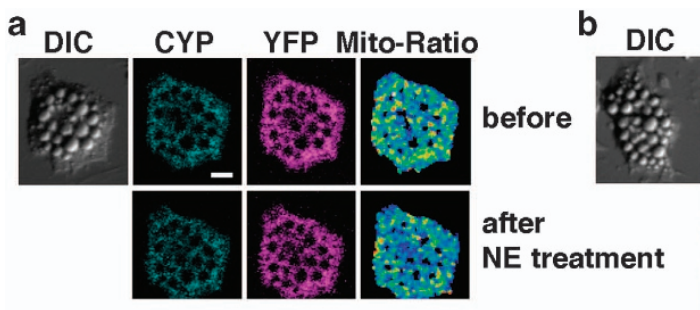
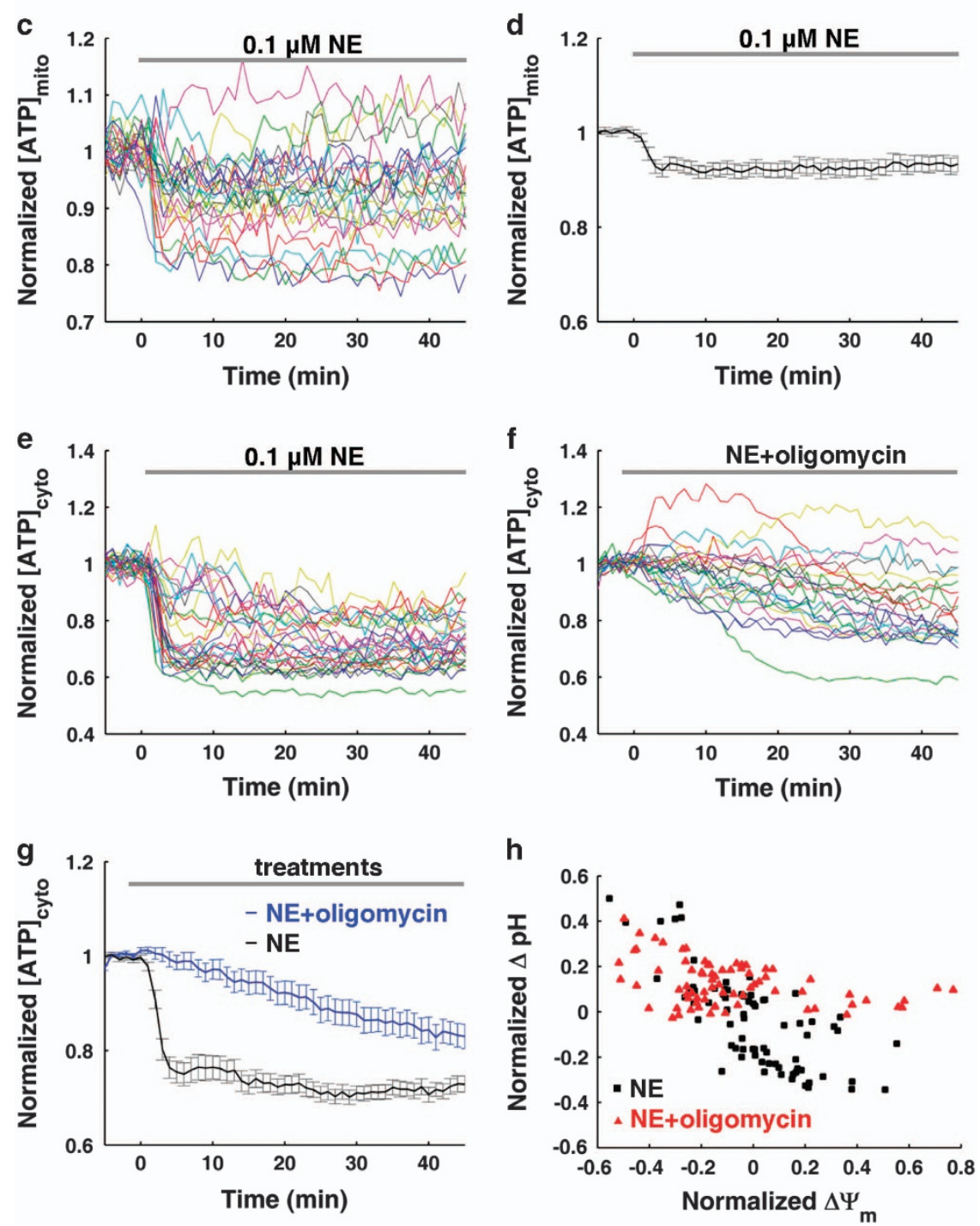

Figure 2. NE stimulates proton-ATPase activity of mitochondrial complex $\mathrm{V}$ in BA. (a-g) Fluorescence of AT1.03-based ratiometric measurements of mitochondrial and cytoplasmic ATP. (a and $\mathbf{b})$ Show fluorescence images of mitAT1.03- and AT1.03-transfected BA before and after $0.1 \mu \mathrm{M}$ NE treatment, respectively. Scale bar, $20 \mu \mathrm{m}$. (c and d) Show raw data plots (c) and an average plot (d) of mitochondrial ATP changes $(n=16)$ in $0.1 \mu \mathrm{M}$ NE-treated BA. Each colored trace represents the ATP change of a single BA (c). (e) Shows the raw data plots of cytoplasmic ATP $(n=29)$ after NE treatment. Each colored trace represents the ATP change of a single BA. (f) Shows the raw data plots of cytoplasmic ATP $(n=23)$ after co-treatment of NE and oligomycin A. Each colored trace represents the ATP change of a single BA. (g) Compares the averaged changes in cytoplasmic ATP after NE treatment $(n=29)$ or NE and oligomycin A co-treatment $(n=23)$, which show that the inhibitor (oligomycin A) of mitochondrial complex $V$ mitigates the ATP consumption induced by NE stimulation. (h) Shows a representative scatter plot of $\mathrm{pH}$ changes versus MMP changes induced by $0.1 \mu \mathrm{M} \mathrm{NE}$ with (red, $n=75$ ) or without (black, $n=75$ ) $10 \mu \mathrm{g} \mathrm{ml}{ }^{-1}$ oligomycin A pretreatment, which demonstrates that the inhibitor of mitochondrial complex $\mathrm{V}$ blocks the activity of the proton pump. All data points in $\mathbf{d}$ and $\mathbf{g}$ represent mean \pm s.e.m. 
thermogenic responses (the fluorescent intensity ratio of Rh800 to RhB-ME) in BA indeed show a negative correlation with mitochondrial electric potential difference $\left(\Delta \Psi_{\mathrm{m}}\right)$ (Figures $1 \mathrm{~d}-\mathrm{f}$ and Supplementary Movies 1-2). Our findings suggest that the heterogeneous responses of MMP in BA can index NE-induced thermogenic efficacy, and they further confirm that the efficacy of NE-induced thermogenesis is low. ${ }^{10}$ Particularly, even a high concentration $(10 \mu \mathrm{M})$ of NE still shows low efficacy with two $\Delta \Psi_{\mathrm{m}}$ populations (Figure 1g) and only slightly increases the thermogenic responses of $\mathrm{BA}$ compared to stimulation with $0.1 \mu \mathrm{M} \mathrm{NE}$ (Figure $1 \mathrm{~h}$ ). However, the NE-induced thermogenic responses of BA are inhibited by $1 \mu \mathrm{M}$ SR-59230A (a potent $\beta$-adrenergic receptor antagonist, Figure $1 \mathrm{~h}$ ).

Considering that mitochondrial $\Delta \Psi_{\mathrm{m}}$ is quasi-linear in physiologically relevant ranges of $\mathrm{pH}$ difference $(\Delta \mathrm{pH}){ }_{1}{ }^{21} \mathrm{MMP}$ hyperpolarization might result from cytoplasmic acidification by enhanced glycolysis or NE-stimulated activities of proton pumps. Indeed, using SNARF1-AM, a ratiometric $\mathrm{pH}$-indicating fluorescent probe, we revealed that the subpopulation of MMP hyperpolarization shows cytoplasmic acidification $(\Delta \mathrm{pH}<0)$ and that $\Delta \Psi_{\mathrm{m}}$ is negatively correlated with $\Delta \mathrm{pH}$ (Figure 1i).

In addition, the phosphorylation level of HSL consistently shows little correlation (Supplementary Figure 3a), whereas the heterogeneity of UCP1 shows a reasonable positive correlation (Supplementary Figure 3b) with NE-induced thermogenesis in BA. Intriguingly, the correlations among NE-induced thermogenic responses, $\Delta \Psi_{\mathrm{m}}, \Delta \mathrm{pH}$ (strong negative correlations, Figures if and i) and UCP1 (moderate positive correlation) suggest that the mitochondrial thermogenesis of BA might be the net result of proton outflow by pumps and proton leakage by UCP1.

NE stimulation activates the proton-ATPase activity of mitochondrial complex $\mathrm{V}$ in $\mathrm{BA}$

Cytoplasmic acidification of MMP-hyperpolarized BA (Figure 1i) can be not only explained by NE-activated proton ATPase $\left(\mathrm{H}^{+}-\right.$ ATPase), but also by enhanced glycolysis. To distinguish the difference and identify the potential roles of enhanced glycolysis (net ATP production) or NE-activated mitochondrial $\mathrm{H}^{+}$-ATPase (ATP consumption) in BA, we next examined the statuses of mitochondrial and cytoplasmic ATP concentrations ([ATP]) after NE stimulation (Figures $2 \mathrm{a}-\mathrm{g}$ ). Using FRET-based ATP indicators (mitAT1.03 and AT1.03), ${ }^{22}$ we found that both cytoplasmic [ATP] and the majority of mitochondrial [ATP] are decreased (Figures $2 \mathrm{C}$ and e) after NE stimulation of BA.

Clearly, these results suggest that NE stimulation activates an ATPase, which is likely a $\mathrm{H}^{+}$-ATPase, that might play a role in the thermogenic efficacy of BA. The best candidate for the $\mathrm{H}^{+}$-ATPase is mitochondrial ATP synthase/complex $V$, which can function as an ATPase in vitro to pump protons. ${ }^{23}$ ATP is consumed by not only mitochondrial ATPase, but also other cellular activities. Therefore, it was necessary to further verify whether complex $V$ is the ATPase activated by NE. Thus, we used oligomycin A, a specific inhibitor of complex $\mathrm{V}$, and revealed that oligomycin $\mathrm{A}$ mitigates the ATP consumption induced by NE stimulation (Figures $2 \mathrm{f}$ and $\mathrm{g}$ ). These results confirmed that NE activates the ATPase function of mitochondrial complex $\mathrm{V}$, which consumes large amounts of ATP assimilating from the cytosol. For complex V-independent ATP consumption, other ATPases, such as a

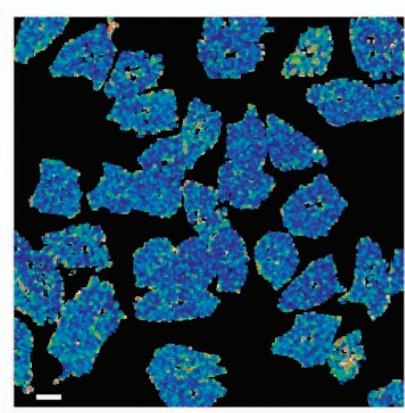

d

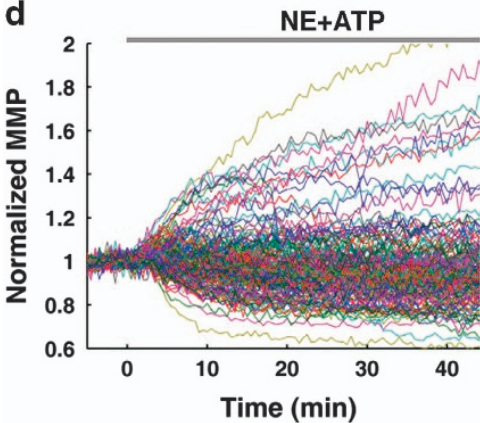

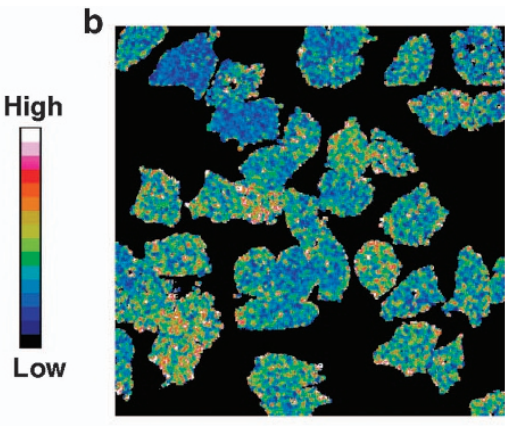

C

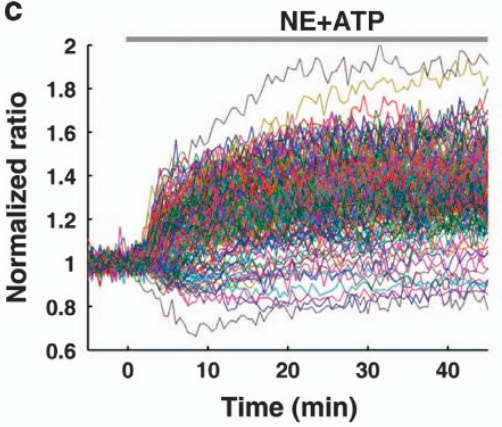

e

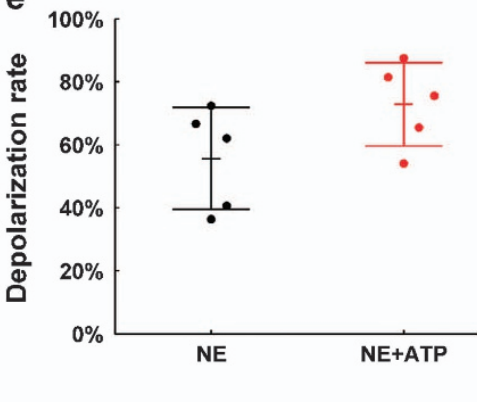

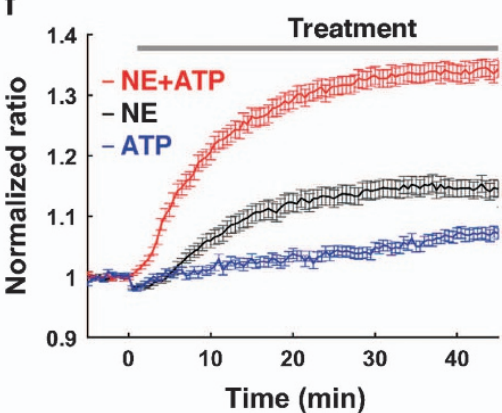

Figure 3. Sympathetic cotransmitter ATP enhances NE-induced thermogenic efficacy in BA. (a and b) Representative thermal images of BA before and after $0.1 \mu \mathrm{M}$ NE and $10 \mu \mathrm{M}$ ATP co-treatment, respectively. Scale bar, $20 \mu \mathrm{m}$. (c) $0.1 \mu \mathrm{M}$ NE and $10 \mu \mathrm{M}$ ATP-induced thermal responses in BA. Each colored trace represents the thermogenic response of a single BA $(n=158)$. (d) The dynamic plots of MMP demonstrate that most BA show MMP depolarization after NE and ATP co-treatment. Each colored trace represents an MMP dynamic of a single BA $(n=158)$. (e) Shows scatter plots of MMP depolarization percentage in $0.1 \mu \mathrm{M}$ NE without (black) or with (red) ATP-treated experiments. NE and ATP co-treatment increases the percentage of MMP depolarization (72.8 $\pm 13.3 \%)$ compared to NE treatment alone (55.6 $\pm 16.1 \%)$. Error bars in the figure represent mean \pm s.d. (f) $0.1 \mu \mathrm{M}$ NE without (black line, also in Figure $1 \mathrm{~h}$ ) or with (red line, $n=158$ ) $10 \mu \mathrm{M}$ ATP-induced thermogenesis in BA. The blue line shows the control results of ATP treatment alone in BA $(n=67)$. All data points in $\mathbf{f}$ represent mean \pm s.e.m. 
adenylate cyclase, could offer an explanation. After NE binds $\beta$ receptors and stimulates the activity of adenylate cyclase via Gs, adenylate cyclase then consumes ATP to produce CAMP, which is necessary for protein kinase $A$ activation.

The role of mitochondrial complex $\mathrm{V}$ as an ATPase in vivo is quite provocative. For example, where does the ATP come from?
The source of ATP production can be provided by NE-stimulated metabolism of glucose because it has been reported and is well known that glucose uptake is increased in BA or BAT by one or two orders of magnitude in human (12-fold) and rat (110 times) under cold stimulation. ${ }^{24,25} \mathrm{NE}$-stimulated metabolism in BA is indeed supported by accelerated metabolic status represented by a
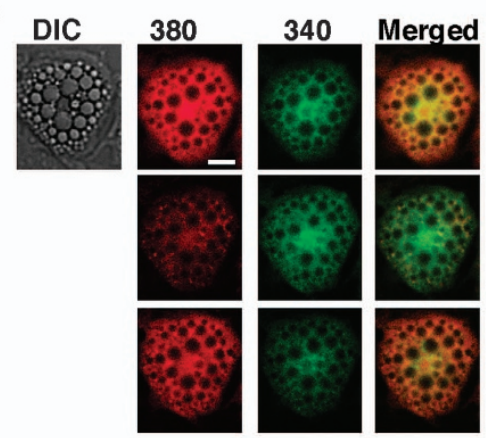

C

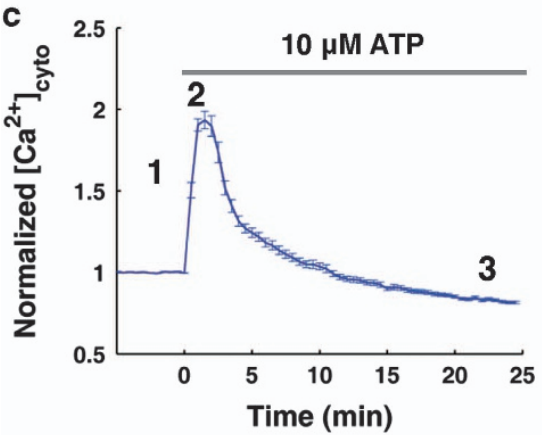

e

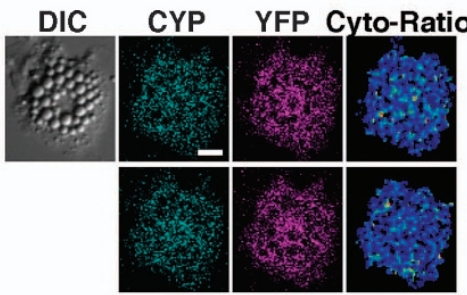

g

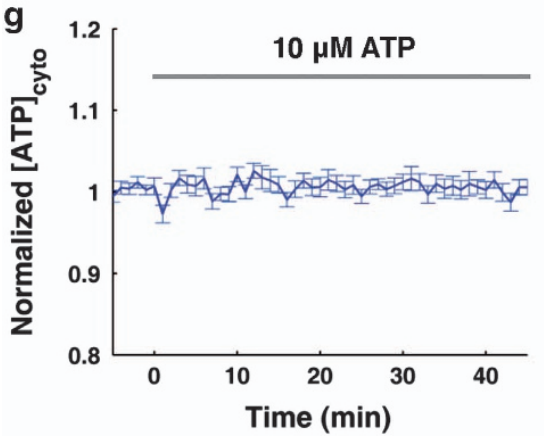

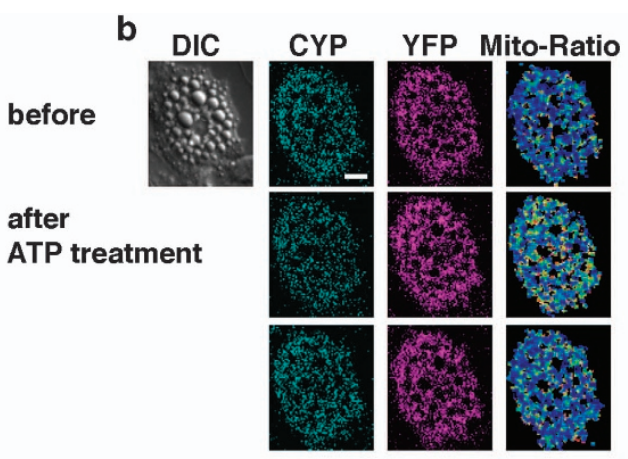
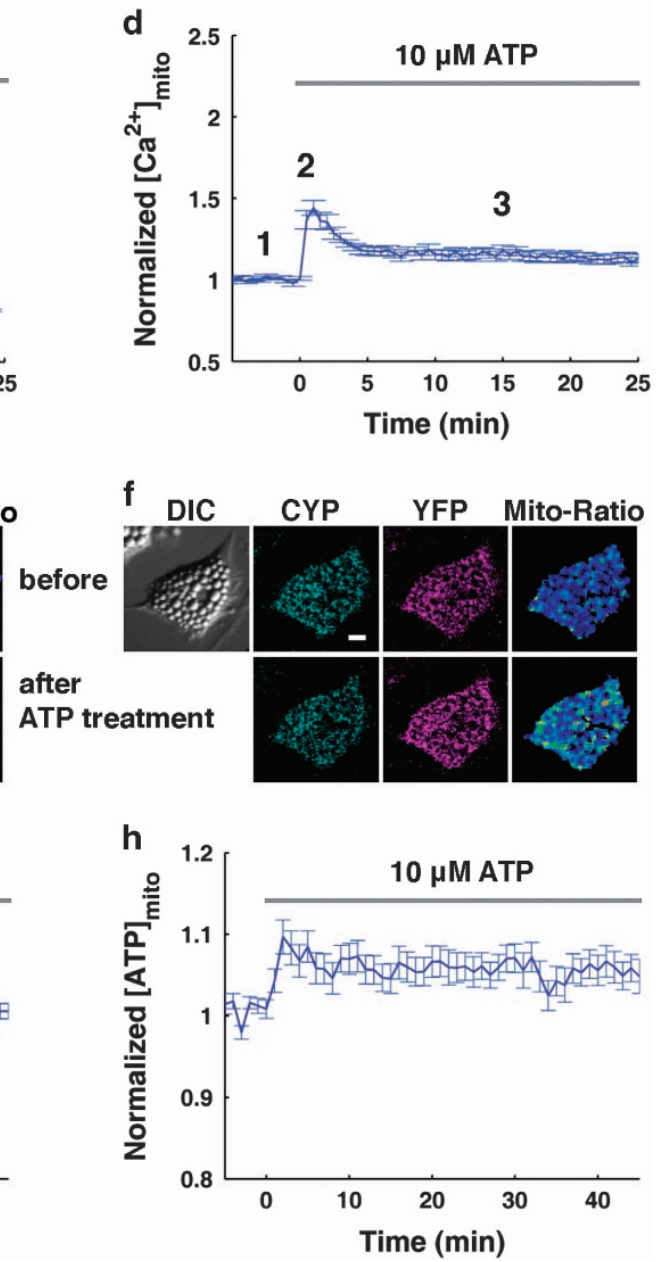

Figure 4. Sympathetic cotransmitter ATP enhances NE-induced thermogenic efficacy in BA by modulating the function of mitochondrial complex V. (a-d) Sympathetic cotransmitter ATP increases cytoplasmic and mitochondrial $\left[\mathrm{Ca}^{2+}\right]$ in BA, which are both fluorescence-based ratiometric measurements. Scale bars, $20 \mu \mathrm{m}$. (a) Shows fluorescence images (excited by 380-nm or 340-nm laser) of Fura2-AM-stained BA before and after $10 \mu \mathrm{M}$ ATP treatment, and positions in c are sequentially labeled with 1,2 and 3. (b) Shows fluorescence images of $4 \mathrm{mtD} 3 \mathrm{cpv}-$ transfected BA before and after $10 \mu \mathrm{M}$ ATP treatment, and positions in $\mathbf{d}$ are sequentially labeled with 1, 2 and 3. (c and d) Show that ATP induces transient elevations of cytoplasmic $(n=63)$ and mitochondrial $(n=14)\left[\mathrm{Ca}^{2+}\right]$ in BA, respectively. (e-h) Fluorescence of AT1.03-based ratiometric measurements of cytoplasmic and mitochondrial ATP concentrations induced by extracellular ATP. (e and f) Show fluorescence images of AT1.03- and mitAT1.03-transfected BA before and after $10 \mu \mathrm{M}$ ATP treatment, respectively. Scale bars, $20 \mu \mathrm{m}$. (g and $\mathbf{h})$ Show the averaged change of cytoplasmic $(n=28)$ and mitochondrial $(n=31)$ ATP by $10 \mu$ M ATP treatment. All data points in $\mathbf{c}$, $\mathbf{d}$, $\mathbf{g}$ and $\mathbf{h}$ represent mean \pm s.e.m. 
homogenous redox changes (Supplementary Figures $4 a-h)$, which are shown in endogenous autofluorescence images and the ratio measurement of $\mathrm{FAD}$ and $\mathrm{NADH} .{ }^{16}$ In addition, as interestingly illustrated in Figure 2f, few BA pretreated with oligomycin A show transiently increased cytoplasmic [ATP], which supports accelerated cytosol metabolism for ATP production after NE stimulation. Moreover, accelerated metabolic status shows a strong positive correlation with NE-induced thermogenesis in BA (Supplementary Figure 5).

To further check whether the ATPase function of complex $V$ causes cytoplasmic acidification after NE stimulation, we used oligomycin $\mathrm{A}$ to inhibit complex $\mathrm{V}$ again and revealed that almost all BA showed cytoplasmic alkalizations $(\Delta \mathrm{pH}>0)$ and that most BA showed MMP depolarization after NE stimulation (Figure $2 \mathrm{~h}$ and Supplementary Figure 6). Thus, these findings further demonstrated and confirmed that NE stimulation activates the $\mathrm{H}^{+}$-ATPase function of complex $\mathrm{V}$ instead of glycolysis for cytoplasmic acidification. Together, our findings suggest that the $\mathrm{H}^{+}$-ATPase activity of complex $\mathrm{V}$ is a key factor in antagonizing proton leakage by UCP1, which impacts the net cytoplasmic $\Delta \mathrm{pH}$ (Figures $1 \mathrm{i}$ and $2 \mathrm{~h}$ ) and modulates the efficacy of NE-induced BA thermogenesis (Supplementary Figure 6b). The efficacy is comparable to CCCP-induced thermogenesis. ${ }^{10}$

\section{ATP as a sympathetic cotransmitter can increase NE-induced thermogenic efficacy}

Our observations suggest that factors other than NE alone are needed for the high efficacy of evoking thermogenesis in BAT. Considering that ATP is also a neurotransmitter and is co-released with NE when sympathetic nerve firing is evoked, ${ }^{26}$ we examined whether ATP is needed as a co-factor for NE to efficiently evoke thermogenesis in BA (Figure 3). As illustrated in Figure 3, most BA showed thermogenic responses (Figures $3 a-c$ ) to NE and ATP COstimulation (Supplementary Movies 3-4). The co-treatment with NE and ATP also increases the percentage (in mean \pm s.d.) of the MMP depolarization subpopulation $(72.8 \pm 13.3 \%)$ in BA compared to NE treatment alone $(55.6 \pm 16.1 \%$ and $P=0.0514$, one-sided $t$ test) (Figures $3 \mathrm{~d}-\mathrm{e}$ ). NE and ATP co-stimulation markedly increased the amplitude of thermogenic responses (Figure $3 \mathrm{f}$ ) in BA compared to NE treatment alone (Supplementary Movies 1-4). In contrast, BA only showed slightly and relative flat responses to ATP treatment alone (Figure 3f).

To elucidate the enhancement effect of sympathetic cotransmitter ATP via purinergic receptors, we evaluated the statuses of cytoplasmic and mitochondrial $\mathrm{Ca}^{2+}$ concentrations $\left(\left[\mathrm{Ca}^{2+}\right]\right)$ with ratiometric probe Fura2-AM and indicator $4 \mathrm{mtD} 3 \mathrm{cpv}^{27}$ respectively (Figure 4 and Supplementary Figure 4). We found that extracellular ATP evokes a transient elevation of cytoplasmic $\left[\mathrm{Ca}^{2+}\right]$ (Figures $4 \mathrm{a}$ and $\mathrm{c}$ ) in BA through multiple $\mathrm{P} 2$ receptors (Supplementary Figure 7 and Supplementary Movie 5), which is in line with previous reports. ${ }^{28}$ In contrast, we found that extracellular ATP evoked transient peaked and steady elevation of mitochondrial $\left[\mathrm{Ca}^{2+}\right]$ (Figures $4 \mathrm{~b}$ and $\mathrm{d}$ ).

Mitochondrial $\left[\mathrm{Ca}^{2+}\right]$ is well recognized as an important control of cellular ATP homeostasis. ${ }^{29}$ Therefore, we examined the states of cytoplasmic and mitochondrial [ATP] after ATP stimulation (Figure $4 \mathrm{e}-\mathrm{h}$ ). We observed a steady elevation of mitochondrial [ATP] and no apparent change of cytoplasmic [ATP] after extracellular ATP stimulation (Figures $4 \mathrm{e}-\mathrm{h}$ ). These findings suggest that sympathetic cotransmitter ATP increases the ATP synthase activity of complex $V$ for ATP production (Figure $4 \mathrm{~h}$ ) by elevating mitochondrial $\left[\mathrm{Ca}^{2+}\right]$ (Figure 4d). Importantly, the thermogenic enhancing function (Figure 3) of ATP as a sympathetic cotransmitter of NE can explain the discrepancy of cold and sympathomimetics activities on human BAT in vivo. ${ }^{30}$

\section{DISCUSSION}

Mitochondrial complex $\mathrm{V}$ is a deterministic factor in NE-induced thermogenic efficacy

How does the $\mathrm{H}^{+}$-ATPase activity of complex $\mathrm{V}$ modulate the thermogenic efficacy of BA? A first straightforward explanation is the endothermic characteristic feature of ATP hydrolysis by mitochondrial complex $V_{1}^{31}$ which pumps dissipated protons back and restores energy in the mitochondrial proton gradient. In Figure 1e, there are some interesting and supporting results, where very few BA show endothermic profiles rather than exothermic profiles in response to NE stimulation. The endothermic profiles could be a stimulus artifact, but this outcome is unlikely. Second, the $\mathrm{H}^{+}$-ATPase activity of complex $\mathrm{V}$ results in cytoplasmic acidification (Figures $1 \mathrm{i}$ and $2 \mathrm{~h}$ ), which can enhance the binding and inhibition of purine nucleotides to UCP $1 .{ }^{32}$

Why does complex $\mathrm{V}$ need to function as an $\mathrm{H}^{+}$-ATPase by NE stimulation? The physiological role of an $\mathrm{H}^{+}$-ATPase function is likely to avoid unnecessary and undesired heat production as NE is either secreted by adrenal medullae or released by the sympathetic nervous system, which can also be excited by mental or physical stress. ${ }^{33}$ Accordingly, for cold stimulation, our observations suggest that at least two factors (NE and ATP) are needed for the high efficacy and specificity of evoking thermogenesis in BAT. Compared with NE+oligomycin or CCCP-induced fully thermogenic responses (Supplementary Figure 6), NE+ATP can utilize $\sim 60 \%$ of the full capacity (Figure 3 ).

In this study, we observed that NE evokes both homogenous responses (redox ratio, cytosol ATP consumption and calcium dynamics) and heterogeneous changes $\left(\Delta \Psi_{\mathrm{m}}, \Delta \mathrm{pH}\right.$ and thermogenesis). Consequently, we have demonstrated that heterogeneous changes and thermogenic efficacy are modulated by the functional status of mitochondrial complex $\mathrm{V}$ and sympathetic transmitters. NE-induced heterogenic responses in BA suggest that it may use a frequency-modulating method for NE-induced mild thermogenesis ( $25 \%$ of thermogenic capacity, Figure $1 \mathrm{~h}$ ). NE co-stimulation with ATP or oligomycin A can evoke thermogenesis in BA with both frequency and amplitude modulations (Figure 3 and Supplementary Figure 6), which are the net results of proton outflow by the $\mathrm{H}^{+}$-ATPase of mitochondrial complex $\mathrm{V}$ and proton leakage by UCP1.

Our findings suggest that mitochondrial malfunctions or impairments in the sympathetic system or BAT can be pathological reasons for inefficient energy expenditure and the development of obesity. Additionally, our current observations also open new doors and pave the way to reconsider targets and strategies that partially or fully utilize the thermogenic capacity of brown or browning $^{34}$ adipocyte for the treatment of obesity, diabetes and abnormal lipid metabolism.

\section{ACKNOWLEDGEMENTS}

We thank the following individuals for their help: $\mathrm{Dr} \mathrm{H}$ Noji and $\mathrm{Dr} \mathrm{H}$ Imamura for the AT1.03 and mitAT1.03 plasmids and Dr R Tsien for the 4mtD3cpv plasmid

\section{AUTHOR CONTRIBUTIONS}

TRX and CFL designed and conducted the experiments and wrote the manuscript. TRX performed live cell imaging and data analysis. CFL performed primary cell culture and imaging. JSK constructed the custom microscopy system, developed the idea, directed the study and wrote the paper. All authors participated in discussions.

\section{COMPETING INTERESTS}

The authors declare no conflict of interest. 


\section{REFERENCES}

1 Lowell BB, Spiegelman BM. Towards a molecular understanding of adaptive thermogenesis. Nature 2000; 404: 652-660.

2 Cannon B, Nedergaard J. Brown adipose tissue: function and physiological significance. Physiol Rev 2004; 84: 277-359.

3 Cypess AM, Lehman S, Williams G, Tal I, Rodman D, Goldfine AB et al. Identification and importance of brown adipose tissue in adult humans. $N$ Engl J Med 2009; 360: 1509-1517.

4 van Marken Lichtenbelt WD, Vanhommerig JW, Smulders NM, Drossaerts JMAFL, Kemerink GJ, Bouvy ND et al. Cold-activated brown adipose tissue in healthy men. N Engl J Med 2009; 360: 1500-1508.

5 Virtanen KA, Lidell ME, Orava J, Heglind M, Westergren R, Niemi T et al. Functional brown adipose tissue in healthy adults. N Engl J Med 2009; 360: 1518-1525.

6 Harms M, Seale P. Brown and beige fat: development, function and therapeutic potential. Nat Med 2013; 19: 1252-1263.

7 Betz MJ, Enerbäck S. Human brown adipose tissue: what we have learned so far. Diabetes 2015; 64: 2352-2360.

8 Nakamura K, Morrison SF. A thermosensory pathway that controls body temperature. Nat Neurosci 2008; 11: 62-71.

9 Fedorenko A, Lishko PV, Kirichok Y. Mechanism of fatty-acid-dependent UCP1 uncoupling in brown fat mitochondria. Cell 2012; 151: 400-413.

10 Xie T-R, Liu C-F, Kang J-S. Dye-based mito-thermometry and its application in thermogenesis of brown adipocytes. Biophys Rep 2017; doi: 10.1007/s41048-0170039-6.

11 Arch JRS. Challenges in $\beta 3$-adrenoceptor agonist drug development. Ther Adv Endocrinol Metab 2011; 2: 59-64.

12 Carey AL, Formosa MF, Every BV, Bertovic D, Eikelis N, Lambert GW et al. Ephedrine activates brown adipose tissue in lean but not obese humans. Diabetologia 2012; 56: 147-155.

13 Lucero MT, Pappone PA. Voltage-gated potassium channels in brown fat cells. J Gen Physiol 1989; 93: 451-472.

14 Fain JN, Reed N, Saperstein R. The isolation and metabolism of brown fat cells. J Biol Chem 1967; 242: 1887-1894.

15 Burchfield JG, Lopez JA, Hughes WE. Using total internal reflection fluorescence microscopy (TIRFM) to visualise insulin action. In: Badoer E (ed). Visualization Techniques. Humana Press: Australia, 2012, pp 97-109.

16 Georgakoudi I, Quinn KP. Optical imaging using endogenous contrast to assess metabolic state. Annu Rev Biomed Eng 2012; 14: 351-367.

17 Sakanoue J, Ichikawa K, Nomura Y, Tamura M. Rhodamine 800 as a probe of energization of cells and tissues in the near-infrared region: a study with isolated rat liver mitochondria and hepatocytes. J Biochem 1997; 121: 29-37.

18 Wikstrom JD, Mahdaviani K, Liesa M, Sereda SB, Si Y, Las G et al. Hormone-induced mitochondrial fission is utilized by brown adipocytes as an amplification pathway for energy expenditure. EMBO J 2014; 33: 418-436.

19 Lafontan M, Berlan M. Fat cell adrenergic receptors and the control of white and brown fat cell function. J Lipid Res 1993; 34: 1057-1091.

20 Cinti S, Cancello R, Zingaretti MC, Ceresi E, Matteis RD, Giordano A et al. CL316,243 and cold stress induce heterogeneous expression of UCP1 mRNA and protein in rodent brown adipocytes. J Histochem Cytochem 2002; 50: 21-31.

21 Schuster S, Ouhabi R, Rigoulet M, Mazat J-P. Modelling the interrelation between the transmembrane potential and $\mathrm{pH}$ difference across membranes with electrogenic proton transport upon build-up of the proton-motive force. Bioelectrochem Bioenerg 1998; 45: 181-192.

22 Imamura H, Huynh Nhat KP, Togawa H, Saito K, lino R, Kato-Yamada $\mathrm{Y}$ et al. Visualization of ATP levels inside single living cells with fluorescence resonance energy transfer-based genetically encoded indicators. Proc Natl Acad Sci USA 2009; 106: 15651-15656.

23 Nicholls D, Fergusion S. Bioenergetics, 3rd edn. Academic Press: London, 2002. http://store.elsevier.com/Bioenergetics/David-Nicholls/isbn-9780080527932/ (accessed 26 Oct 2016).

24 Orava J, Nuutila P, Lidell ME, Oikonen V, Noponen T, Viljanen T et al. Different metabolic responses of human brown adipose tissue to activation by cold and insulin. Cell Metab 2011; 14: 272-279.

25 Vallerand AL, Perusse F, Bukowiecki LJ. Stimulatory effects of cold exposure and cold acclimation on glucose uptake in rat peripheral tissues. Am J Physiol Regul Integr Comp Physiol 1990; 259: R1043-R1049.

26 Burnstock G. Historical review: ATP as a neurotransmitter. Trends Pharmacol Sci 2006; 27: 166-176.

27 Palmer AE, Tsien RY. Measuring calcium signaling using genetically targetable fluorescent indicators. Nat Protoc 2006; 1: 1057-1065.

28 Omatsu-Kanbe $\mathrm{M}$, Isono $\mathrm{T}$, Matsuura $\mathrm{H}$. Multiple P2 receptors contribute to a transient increase in intracellular $\mathrm{Ca} 2+$ concentration in ATP-stimulated rat brown adipocytes. Exp Physiol 2002; 87: 643-652.

29 Tarasov Al, Griffiths EJ, Rutter GA. Regulation of ATP production by mitochondrial Ca2+. Cell Calcium 2012; 52: 28-35.

30 Cypess AM, Chen Y-C, Sze C, Wang K, English J, Chan O et al. Cold but not sympathomimetics activates human brown adipose tissue in vivo. Proc Natl Acad Sci USA 2012; 109: 10001-10005.

31 Dittrich M, Hayashi S, Schulten K. On the mechanism of ATP hydrolysis in F1ATPase. Biophys J 2003; 85: 2253-2266.

32 Rafael J, Pampel I, Wang X. Effect of $\mathrm{pH}$ and $\mathrm{MgCl} 2$ on the binding of purine nucleotides to the uncoupling protein in membrane particles from brown fat mitochondria. Eur J Biochem 1994; 223: 971-980.

33 Guyton A, Hall J. Textbook of Medical Physiology, 11th edn. Elsevier Saunders: Philadelphia, 2006. http://www.us.elsevierhealth.com/physiology/ textbook-of-medical-physiology-hardcover/9780721602400/ (accessed 22 Feb 2016).

34 Than A, Xu S, Li R, Leow M-S, Sun L, Chen P. Angiotensin type 2 receptor activation promotes browning of white adipose tissue and brown adipogenesis. Signal Transduct Target Ther 2017; 2: 17022.

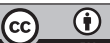

This work is licensed under a Creative Commons Attribution 4.0 International License. The images or other third party material in this article are included in the article's Creative Commons license, unless indicated otherwise in the credit line; if the material is not included under the Creative Commons license, users will need to obtain permission from the license holder to reproduce the material. To view a copy of this license, visit http://creativecommons.org/licenses/ by/4.0/

(c) The Author(s) 2017

Supplementary Information accompanies the paper on the Signal Transduction and Targeted Therapy website (http://www.nature.com/sigtrans) 